See discussions, stats, and author profiles for this publication at: https://www.researchgate.net/publication/260148239

\title{
Biohumoral markers as predictor of right ventricular dysfunction in $\mathrm{AL}$ Amyloidosis
}

Article in Amyloid: the international journal of experimental and clinical investigation: the official journal of the International Society of Amyloidosis · February 2014 DOI: 10.3109/13506129.2014.884971· Source: PubMed

CITATIONS

8

13 authors, including:

Francesco Cappelli

Azienda Ospedaliero Universitaria Careggi

92 PUBLICATIONS 1,177 CITATIONS

SEE PROFILE

Luigi Padeletti

University of Florence

542 PUBLICATIONS 10,858 CITATIONS

SEE PROFILE
READS

74

Franco Bergesio

Azienda Ospedaliero Universitaria Careggi

94 PUBLICATIONS 908 CITATIONS

SEE PROFILE

Paola Attanà

Azienda Unità Sanitaria Locale 10 Firenze

47 PUBLICATIONS 480 CITATIONS

SEE PROFILE

Some of the authors of this publication are also working on these related projects:

amyloidosis View project

Project Response to Cardiac Resynchronization Therapy View project 


\title{
Biohumoral markers as predictor of right ventricular dysfunction in AL Amyloidosis
}

\author{
Francesco Cappelli ${ }^{1,2}$, Samuele Baldasseroni ${ }^{1}$, Franco Bergesio ${ }^{2}$, Luigi Padeletti ${ }^{3}$, Paola Attanà ${ }^{3}$, \\ Alberto Moggi Pignone ${ }^{4}$, Elisa Grifoni ${ }^{4}$, Gabriele Ciuti ${ }^{4}$, Alessia Fabbri ${ }^{4}$, Francesca Tarantini ${ }^{5}$, Niccolò Marchionni ${ }^{5}$, \\ Gian Franco Gensini ${ }^{3}$, and Federico Perfetto ${ }^{2}$ \\ ${ }^{1}$ Department of Heart and Vessel, Intensive Cardiac Care Unit, AOUC, Florence, Italy, ${ }^{2}$ Regional Amyloid Center, AOUC, Florence, Italy, \\ ${ }^{3}$ Department of Heart and Vessels, ${ }^{4}$ Department of Internal Medicine, and ${ }^{5}$ Department of Clinical and Experimental Medicine, \\ University of Florence, Florence, Italy
}

\begin{abstract}
Aim: In AL amyloidosis, the importance of right ventricle (RV) involvement has recently been underlined and its role in predicting prognosis has been emphasized. Little is known about the relationship between RV involvement, N-terminal pro-brain natriuretic peptide (NT-proBNP) and troponin levels. Aim of our study was to clarify the relationship between NT-proBNP and troponin and RV involvement and analyze their independent value as predictors of RV dysfunction.

Methods and Results: We examined 76 consecutive patients with biopsy-proven AL amyloidosis. Each patient received complete clinical evaluation, troponin I, NT-proBNP assay and comprehensive echocardiographic evaluation. Considering a tricuspidal annulus plane systolic excursion (TAPSE) value $<16 \mathrm{~mm}, 23$ patients (30\%) presented RV systolic dysfunction, whereas $53(70 \%)$ did not. Patient with reduced TAPSE had thicker left ventricle (LV) walls and RV free walls, reduced LV fractional shortening, impaired LV diastolic function and worse LV and RV myocardial performance index. For RV dysfunction the best predictive value for NT-proBNP was identified as $2977 \mathrm{ng} / \mathrm{l}$ with sensitivity and specificity of $87 \%$ and $84 \%$, respectively; best cut-off for troponin I was identified as $0.085 \mathrm{ng} / \mathrm{l}$, with sensitivity and specificity of $85 \%$ and $90 \%$ respectively.

At multivariable logistic regression analysis, both NT-proBNP and troponin I emerged as independent predictors of RV dysfunction presence but troponin appears to have a higher predictive power.

Conclusion: Our study demonstrated that cut-off values of $2977 \mathrm{ng} / \mathrm{ml}$ for NT-proBNP and $0.085 \mathrm{ng} / \mathrm{l}$ for troponin were able to identify a subgroup of AL patients with RV dysfunction. Troponin I is more accurate and seems to be the best biohumoral marker of RV dysfunction.
\end{abstract}

\begin{abstract}
Abbreviations: BSA: body surface area; DT: E wave deceleration time; EF: LV ejection fraction; FLC: free light chain; FS: LV fractional shortening; IVS: end-diastolic thickness of interventricular septum; LV: left ventricular; LV EDD and LV ESD: LV end-diastolic and end-systolic diameters respectively; LV EDV and LV ESV: LV end-diastolic and end-systolic volumes respectively; MPI: myocardial performance index; MDRD: modification of diet renal disease; NT-proBNP: $\mathrm{N}$-terminal pro-brain natriuretic peptide; PASP: pulmonary artery systolic pressure; PW: LV posterior wall; RV: right ventricle; RV EDD: RV end diastolic diameter; TAPSE: tricuspidal annulus plane systolic excursion; TDI: tissue Doppler imaging
\end{abstract}

\section{Introduction}

Amyloidosis is a rare systemic disease characterized by extracellular deposition of protein derived fibrils in various tissues and organs, including the heart [1-2]. Cardiac

Address for correspondence: Francesco Cappelli MD, Department of Heart and Vessels, Intensive Cardiac Unit, University of Florence and Azienda Ospedaliero-Universitaria Careggi [AOUC], Largo Brambilla 3, Florence 50134, Italy. Tel: +39 557947518. Fax: +39 557947706. E-mail: cappellifrancesco@inwind.it

\section{Keywords}

AL amyloidosis, NT-proBNP, right ventricular dysfunction, troponin

\section{History}

Received 4 July 2013

Revised 24 November 2013

Accepted 9 January 2014

Published online 11 February 2014 involvement usually determines prognosis and, among the different forms of cardiac amyloidosis, Amyloid Light-chain (AL) has the worse outcome [3]. Since the hallmark of cardiac amyloidosis is a restrictive cardiomyopathy, medical attention has been focused traditionally on left ventricular (LV) diastolic function. However, in the last few years, due to the availability of more advanced echocardiographic techniques, such as tissue Doppler imaging (TDI) and 2-dimensional speckle tracking echocardiography, consideration has been given to the study of geometrical modification of LV systolic 
function [4-6]. In addition, the involvement of the right ventricle (RV) and its role in predicting prognosis in $\mathrm{AL}$ amyloidosis has recently been emphasized [7-8]. Since the seminal work of Paladini et al. [9], N-terminal pro-brain natriuretic peptide (NT-proBNP) and troponin have been employed in AL amyloidosis for diagnosis of cardiac involvement and prognostic evaluation [10]. Although several studies have proven the relationship between cardiac biomarkers and RV dysfunction in patients with acute pulmonary embolism and arterial pulmonary hypertension [11,12], comparison among those cardiac biomarkers in the prediction of RV dysfunction and their optimal cutoff values in patients with cardiac AL amyloidosis were poorly investigated.

So the aims of our study were (1) to clarify the relationship between NT-proBNP and troponin and RV involvement (2) to analyze the independent value of NT-proBNP and troponin as indicators of RV dysfunction and identify the best cut off values.

\section{Material and methods}

Seventy-six consecutive patients (43 females and 33 males, mean age $69.5 \pm 9.8$ years) with biopsy-proven AL amyloidosis were examined at the Tuscan Regional Centre of Amyloidosis, Florence (Italy), between February, 2007, and December, 2012. All participants gave informed written consent, following the Helsinki declaration. Positive biopsy site was abdominal fat in 52 patients (68\%), kidney in 12 (17\%), myocardium in $4(5 \%)$, salivary gland in $3(4 \%)$, bone marrow in 2 (3\%), stomach, lymph node and liver in 1 patient (1\%). All positive biopsies demonstrated the typical Congo red birefringence under polarized light. According to international criteria [13], AL amyloidosis was confirmed by the finding of a monoclonal protein in the serum or urine and/or a monoclonal population of plasma cells in the bone marrow by immunohistochemistry. All participants underwent a thorough medical history and a complete clinical evaluation. Hereditary amyloidosis was excluded by DNA analysis in all patients.

NT-proBNP was measured with an electro-chemiluminescence sandwich immunoassay (ECLIA, Roche Diagnostic, Indianapolis, IN) in the central hospital laboratory. Troponin I measurements were performed by immuno-chemiluminescence assay using a Centaur XP (Siemens Healthcare, Erlangen, Germany). Serum free light chains (FLC) were determined using the Freelite assay (Binding Site, Birmingham, United Kingdom). Creatinine clearance using modification of diet renal disease (MDRD) formula [14] and both systolic and diastolic arterial blood pressure were evaluated.

\section{Standard and TDI echocardiography}

At the time of diagnosis patients were referred to our laboratory for M-mode, 2-dimensional, conventional and tissue Doppler echocardiographic study [15]. Echocardiography was performed by a single experienced operator (FC), blinded to the clinical history of the patient, using a Vivid 7 System (Vingmed, General Electric, Horten, Norway) equipped with a $3 \mathrm{~S}$ probe. At least three consecutive beats were recorded and the images were digitized and analyzed off-line. The following parameters were assessed: end-diastolic thickness of interventricular septum (IVS) and LV posterior wall (PW), LV end-diastolic and end-systolic diameters (LV EDD and LV ESD, respectively), body surface area (BSA)-indexed LV mass (LVmassind), LV endocardial fractional shortening (FS), left atrial area (LAA, evaluated from the apical four chamber view at the end of systole), LV end-diastolic and end-systolic volumes (LV EDV and LV ESV, respectively), ejection fraction (EF, estimated with the biplane Simpson method), mitral peak flow velocity in early and late diastole (E and A, respectively, during atrial contraction), E wave deceleration time (DT), E/A ratio, LV and RV myocardial performance index (MPI, the sum of isovolumic contraction and relaxation times divided by ejection time, as previously described [16]), $\mathrm{RV}$ free wall thickness (RV FW), RV end-diastolic diameter (RV EDD, evaluated from parasternal long axis view) and the systolic displacement of the lateral portion of the tricuspid annular plane (TAPSE).

Pulmonary artery systolic pressure (PASP) was approximated by adding to trans-tricuspid pressure gradient an estimate of right atrial pressure. We also evaluated pulsed TDI-derived early diastolic peak velocity at mitral annulus (E'), as an index of LV relaxation, and E/E' ratio as an index of LV filling pressure. According to the literature, patients were classified as having RV dysfunction when TAPSE value was $<16 \mathrm{~mm}$ [17].

\section{Statistical analysis}

Statistical analysis was conducted using the $\operatorname{SPSS}^{\circledR}$ for Windows package version 13 (SPSS Inc., Chicago, IL). Categorical and continuous variables were expressed as frequencies (percentages) and as mean \pm standard deviation, respectively. Categorical comparisons were performed with Pearson Chi square and one factor analysis of variance (ANOVA) was used for comparison between groups. Area under the receiver operating characteristics curve (ROC) analysis was used to determine the optimal cutoff of troponin and NT-proBNP as markers of RV dysfunction; sensitivity, specificity, positive and negative predicted value were computed. Among variables that had an independent predictive value on the presence or absence of RV dysfunction at univariate analysis, but with similar clinical significance, only one was introduced in the multivariate logistic regression model, to avoid co-linearity. On this basis we tested three different multivariable logistic models in which NT-proBNP (Model 1), troponin I (Model 2) e NT-proBNP + troponin I (Model 3) were introduced as independent variables. A $p$ value less than 0.05 was considered statistically significant.

\section{Results}

Clinical and echocardiographic characteristics of the study population are reported in Tables 1 and 2. According to a TAPSE value $<16 \mathrm{~mm}, 23$ patients $(30 \%)$ were classified as having a RV systolic dysfunction; 53 (70\%) had a normal RV function. As reported in Table 1, no differences were observed between normal and dysfunctional patients in terms of age, gender, eGFR and number of AL amyloidosis involved organs. The prevalence of a history of diabetes mellitus, arterial hypertension, coronary artery disease and peripheral artery disease did not differ between the groups (48\% in 
Table 1. Demographic. clinical and biohumoral characteristics.

\begin{tabular}{|c|c|c|c|c|}
\hline & All patients & $\begin{array}{c}\text { TAPSE } \geq 16 \mathrm{~mm} \\
(n=53)\end{array}$ & $\begin{array}{c}\text { TAPSE }<16 \mathrm{~mm} \\
(n=23)\end{array}$ & $p$ Value \\
\hline Age (years) & $69.5 \pm 9.8$ & $68.9 \pm 10.1$ & $70.7 \pm 9.2$ & 0.49 \\
\hline Gender $(\mathrm{M} / \mathrm{F})$ & $33 / 43$ & $24 / 29$ & $9 / 14$ & 0.61 \\
\hline $\operatorname{BSA}\left(\mathrm{m}^{2}\right)$ & $1.7 \pm 0.3$ & $1.7 \pm 0.3$ & $1.6 \pm 0.4$ & 0.53 \\
\hline eGFR (ml/min) & $58.7 \pm 26.5$ & $58.3 \pm 28.4$ & $59.8 \pm 21.7$ & 0.83 \\
\hline Arterial diastolic pressure $(\mathrm{mm} / \mathrm{Hg})$ & $69 \pm 11$ & $70 \pm 9$ & $69 \pm 10$ & 0.18 \\
\hline Arterial sistolic pressure $(\mathrm{mm} / \mathrm{Hg})$ & $117 \pm 16$ & $119 \pm 16$ & $115 \pm 19$ & 0.12 \\
\hline NT-proBNP(ng/l) & $5177.6 \pm 9113$ & $2132 \pm 4972$ & $11797 \pm 12240$ & 0.001 \\
\hline Troponin I(ng/) & $0.12 \pm .21$ & $0.039 \pm .035$ & $0.28 \pm .31$ & 0.001 \\
\hline Free light $\mathrm{K} / \lambda \%$ & $23 / 53$ & $20 / 33$ & $3 / 20$ & 0.06 \\
\hline \multicolumn{5}{|l|}{ NYHA Class } \\
\hline I-II & 60 & 49 & 11 & \multirow[t]{2}{*}{0.0001} \\
\hline III-IV & 16 & 4 & 12 & \\
\hline \multicolumn{5}{|l|}{ Number of involved organs } \\
\hline I & 45 & 33 & 12 & \multirow[t]{4}{*}{0.43} \\
\hline II & 21 & 12 & 9 & \\
\hline III & 6 & 5 & 1 & \\
\hline IV & 4 & 2 & 2 & \\
\hline
\end{tabular}

Table 2. Echocardiographic characteristic of studied population.

\begin{tabular}{|c|c|c|c|c|}
\hline & All patients & $\begin{array}{c}\text { TAPSE } \geq 16 \mathrm{~mm} \\
(n=53)\end{array}$ & $\begin{array}{c}\text { TAPSE }<16 \mathrm{~mm} \\
(n=23)\end{array}$ & $p$ Value \\
\hline LVDD $(\mathrm{mm})$ & $44.8 \pm 6.1$ & $45.5 \pm 4.8$ & $43 \pm 8.2$ & 0.14 \\
\hline LVSD (mm) & $27.6 \pm 5.8$ & $26.9 \pm 5.3$ & $29.3 \pm 6.8$ & 0.1 \\
\hline FS $\%$ & $38.3 \pm 8.8$ & $40.9 \pm 8.4$ & $32.4 \pm 6.7$ & 0.0001 \\
\hline IVS (mm) & $12.9 \pm .2$ & $11.5 \pm 2$ & $16.3 \pm 3.1$ & 0.0001 \\
\hline $\mathrm{PW}(\mathrm{mm})$ & $12.8 \pm 3.0$ & $11.5 \pm 2.3$ & $15.7 \pm 2.3$ & 0.0001 \\
\hline LV mass index $\left(\mathrm{g} / \mathrm{m}^{2}\right)$ & $131.3 \pm 3.1$ & $113 \pm 33$ & $171 \pm 36$ & 0.0001 \\
\hline RV FW (mm) & $6.4 \pm 1.3$ & $5.9 \pm 1.2$ & $7.4 \pm 0.7$ & 0.0001 \\
\hline RVDD (mm) & $28.3 \pm 6.5$ & $27.3 \pm 6.4$ & $30.3 \pm 6.5$ & 0.07 \\
\hline LVEDV (ml) & $80.2 \pm 23.8$ & $83.5 \pm 19.9$ & $72.8 \pm 29.9$ & 0.07 \\
\hline LVESV (ml) & $33.1 \pm 14$ & $33.6 \pm 12.8$ & $31.7 \pm 16.5$ & 0.58 \\
\hline $\mathrm{EF}(\%)$ & $59 \pm 8.5$ & $59.9 \pm 8.3$ & $56.9 \pm 8.9$ & 0.16 \\
\hline LA area $\left(\mathrm{cm}^{2}\right)$ & $20.9 \pm 4.6$ & $19.3 \pm 3.9$ & $24.4 \pm 4.3$ & 0.0001 \\
\hline Mitral Regurgitation mild -moderate/Severe & $66 / 10$ & $50 / 3$ & $16 / 7$ & 0.003 \\
\hline $\mathrm{E}(\mathrm{cm} / \mathrm{s})$ & $74.9 \pm 20.2$ & $72.4 \pm 18.6$ & $80.5 \pm 22.8$ & 0.11 \\
\hline $\mathrm{A}(\mathrm{cm} / \mathrm{s})$ & $69.8 \pm 27.0$ & $78.6 \pm 24.7$ & $49.1 \pm 20.5$ & 0.0001 \\
\hline $\mathrm{E} / \mathrm{A}$ & $1.3 \pm .83$ & $1 \pm 0.6$ & $1.9 \pm 0.9$ & 0.0001 \\
\hline DT (ms) & $207 \pm 76$ & $219 \pm 75$ & $180 \pm 74$ & 0.045 \\
\hline$E^{\prime}(\mathrm{cm} / \mathrm{s})$ & $5.8 \pm 2$ & $6.5 \pm 2$ & $4.4 \pm 1.1$ & 0.0001 \\
\hline $\mathrm{E} / \mathrm{E}$ & $14.3 \pm 7$ & $12 \pm 5.3$ & $19.3 \pm 7.9$ & 0.0001 \\
\hline LV MPI & $0.46 \pm .20$ & $0.38 \pm 0.15$ & $0.63 \pm 0.19$ & 0.0001 \\
\hline PASP $(\mathrm{mm} / \mathrm{Hg})$ & $33.2 \pm 11.8$ & $28.5 \pm 9.7$ & $41.5 \pm 10.9$ & 0.0001 \\
\hline RV MPI & $0.39 \pm .23$ & $0.34 \pm 0.16$ & $0.48 \pm 0.3$ & 0.04 \\
\hline
\end{tabular}

LV: left ventricular; RV: right ventricular; IVS: interventricular septum thickness; PW: posterior wall thickness; RWFW: RV free wall thickness, RVDD: RV end diastolic diameter LVEDD: LV end-diastolic diameter; LVESD: LV end-systolic diameter; FS: Fractional Shortening; LA: Left Atrium; LVEDV: LV end-diastolic volume LVESV: LV end-systolic volume; EF: Ejection Fraction; E: early diastolic mitral peak flow velocity; A: late diastolic mitral peak flow velocity; E': early diastolic peak velocity at lateral mitral annulus; MPI: Myocardial performance index; PASP: pulmonary artery systolic pressure; TAPSE: Tricuspid annular plane systolic excursion

patients with RV systolic dysfunction and $51 \%$ in patient without RV systolic dysfunction). Patients with reduced RV function had more frequent heart failure symptoms and higher New York Heart Association (NYHA) class; they also had higher NT-proBNP and troponin I plasma values.

Patients with reduced TAPSE had thicker LV PW, higher

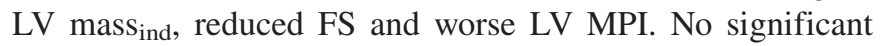
differences in LV systolic and diastolic volumes and diameters were found while atrial area was significantly larger in patients with RV dysfunction (Table 2). As for diastolic function, patients with RV dysfunction showed lower A velocity and higher E/A ratio, with significantly reduced E' and increased E/E' ratio. E velocity and DT were similar between groups. Patients with reduced TAPSE had thicker RV FW with comparable RV EDD, enhanced RV MPI and a significant increase in PASP.

\section{Biohumoral predictors of RV dysfunction}

As reported in Figure 1(A), the best predictive value for NTproBNP was $2977 \mathrm{ng} / \mathrm{l}$, with an area under the curve of 0.90 $(p<0.0001)$ and a sensitivity and specificity of $87 \%$ and $84 \%$, respectively. Using the prevalence of RV involvement observed in our cohort (35\%), the positive and negative 

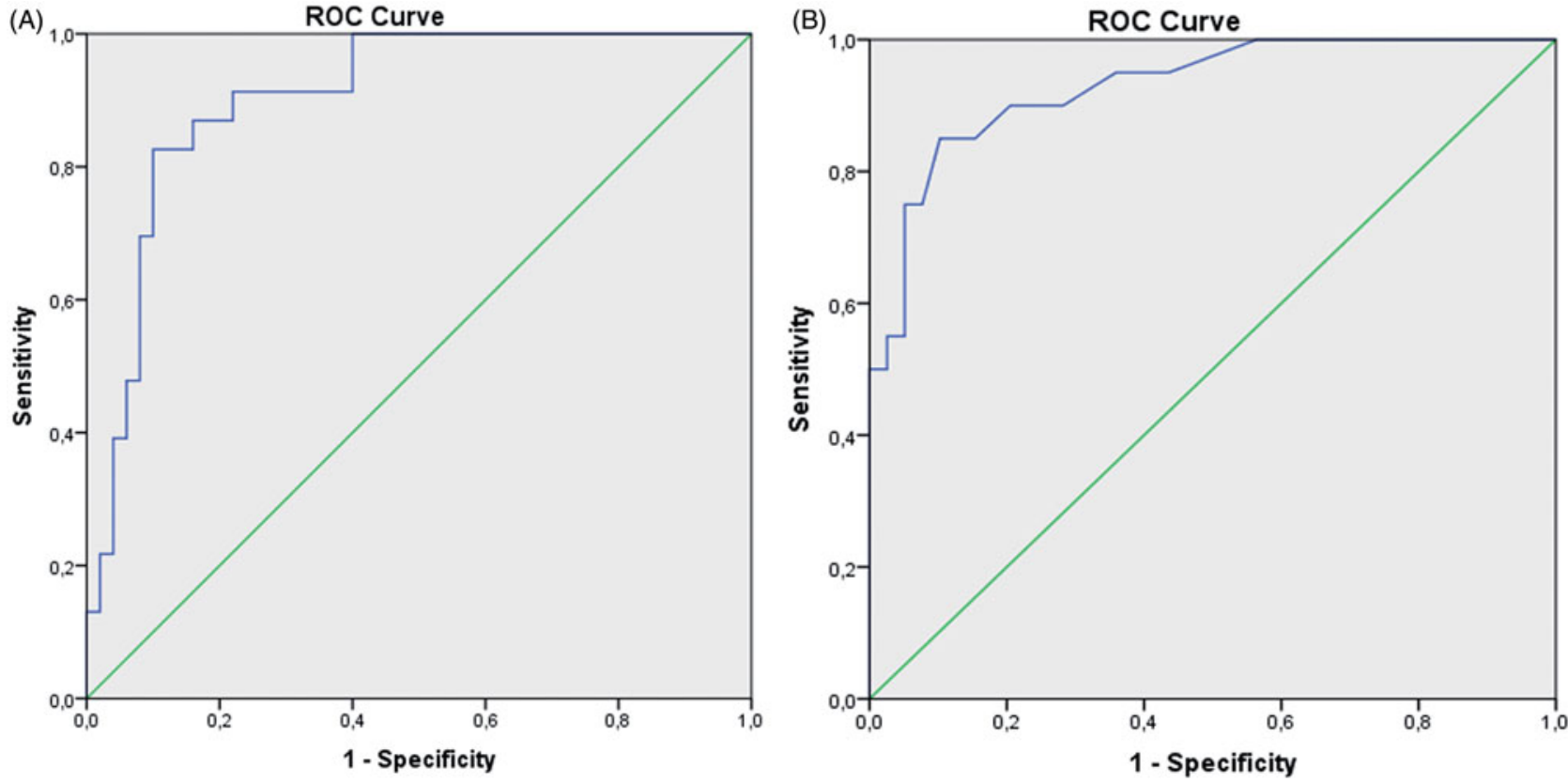

Figure 1. (A) The receiver-operator characteristic analysis for the study population indicated that NT-proBNP with a cut off value of $2977 \mathrm{ng} / \mathrm{l}$ reached a sensitivity of $87 \%$ and specificity of $84 \%$ in detecting right ventricular dysfunction AUC $0.90 p<0.0001$. (B) The receiver-operator characteristic analysis for the study population indicated that Troponin I with a cut off value of $0.085 \mathrm{ng} / \mathrm{l}$ reached a sensitivity of $85 \%$ and specificity of $90 \%$ in detecting right ventricular dysfunction AUC $0.93 p<0.0001$.

Table 3. Multivariable logistic regression analysis models.

\begin{tabular}{|c|c|c|c|c|c|c|c|c|c|}
\hline & \multicolumn{3}{|c|}{ Model 1} & \multicolumn{3}{|c|}{ Model 2} & \multicolumn{3}{|c|}{ Model 3} \\
\hline Age & $0.04 \pm 0.04$ & 1.1 & 0.28 & $0.20 \pm 0.10$ & 3.77 & 0.06 & $0.19 \pm 0.11$ & 2.8 & 0.09 \\
\hline LV mass index & $0.036 \pm 0.013$ & 10.2 & 0.001 & $0.06 \pm 0.03$ & 4.3 & 0.04 & $0.06 \pm 0.03$ & 4.3 & 0.04 \\
\hline FS\% & $-0.07 \pm 0.05$ & 2 & 0.15 & $-0.08 \pm 0.09$ & 0.76 & 0.38 & $-0.83 \pm 0.09$ & 0.76 & 0.38 \\
\hline Mitral Regurgition & $-1.75 \pm 0.9$ & 3.6 & 0.06 & $-3.5 \pm 1.7$ & 3.95 & 0.05 & $-3.5 \pm 1.7$ & 3.9 & 0.05 \\
\hline Troponin I & & & & $98.3 \pm 46$ & 4.46 & 0.03 & $98 \pm 46$ & 4.4 & 0.03 \\
\hline
\end{tabular}

predictive values were $62.5 \%$ and $97.5 \%$, respectively. Troponin I best cut-off was $0.085 \mathrm{ng} / \mathrm{l}$ with an area under the curve of $0.93(p<0.0001)$, a sensitivity of $85 \%$ and a specificity of $90 \%$ (Figure 1B); positive and negative predictive values were $75 \%$ and $88.2 \%$, respectively.

Nine patients showed NT-proBNP concentrations above the cut-off value ( $2977 \mathrm{ng} / \mathrm{l})$ but did not exhibit RV dysfunction (false positive); four of them also had troponin I serum levels above $0.085 \mathrm{ng} / \mathrm{l}$. Eight out of nine had high serum creatinine levels with MDRD lower than $50 \mathrm{ml} / \mathrm{min}$. The results of the three multivariable logistic models are reported in Table 3: both NT-proBNP and troponin I emerged as independent predictors of RV dysfunction (Model 1 and Model 2), with troponin I being the strongest predictor (Model 3).

\section{Discussion}

We studied and compared the predictive value of two commonly used cardiac biomarkers, NT-proBNP and troponin I, in evaluating the presence of RV dysfunction, as determined by echocardiography, in AL cardiac amyloidosis patients. We found that troponin I showed a better sensitivity and specificity than NT-proBNP.

Several reports have demonstrated that circulating NT-proBNP and troponin are valuable markers of cardiac involvement in $\mathrm{AL}$ amyloidosis and are predictors of overall survival [9, 18-21]. The clinical contribution of RV dysfunction in AL cardiac amyloidosis has been emphasized previously $[7,8,22]$. At present, echocardiography remains the best method for the study of RV function and a dysfunctional $\mathrm{RV}$, assessed by different echocardiographic techniques, is an independent, useful predictor of mortality in cardiac AL amyloidosis patients [7,8,22,23].

Recently, our group demonstrated that a mean RV longitudinal 2D speckle-tracking strain less than $-17 \%$ identifies a subgroup of cardiac AL amyloidosis patients with marked RV dysfunction and high risk of death [8]. In addition, at multivariate analysis, RV longitudinal strain was the only echocardiographic predictor of prognosis [8]. These data were in agreement to what was reported by Ghio and coworkers that identified TAPSE as a marker 
of RV systolic dysfunction and a predictor of poor prognosis [18].

Thus, an early identification of RV dysfunction in such patients might provide therapeutic guidance because such patients need rapidly active treatment but at the same time they are extremely fragile and sensitive to treatment toxicity. Despite the fact that echocardiographic evaluation of RV function, as with TAPSE, is feasible even with low technology, widespread echocardiographic equipments, determining NT-proBNP and troponin I serum concentrations could be even easier in every day clinical practice. However, in order to do that, the best cut-off values of the two biomarkers need to be determined. To our knowledge this is the first study that attempted to answer this question.

Using a cohort of patients with cardiac AL amyloidosis we found that both NT-proBNP and troponin I was able to predict $\mathrm{RV}$ involvement. Interestingly, in our cohort the value of NT-proBNP and troponin I was, on average, higher than what was previously reported to be correlated with a generic "amyloid heart involvement"' $[9,10,20]$.

Indeed, the best predictive value of NT-proBNP for RV amyloid dysfunction was $2977 \mathrm{ng} / \mathrm{l}$ which was above the mean value usually indicative of overt heart failure [24]. However, amyloidosis is an infiltrative disease that affects both ventricles walls and which exerts its deleterious effects on the heart also through a direct toxic action of the amyloidogenic free light chains. We can speculate that this high cut off value could be the consequence of LV dysfunction and direct RV infiltration as suggested by increased RV free wall thickness.

These data also confirms the previous observations that in $\mathrm{AL}$ amyloidosis patients, RV dysfunction occurs later than $\mathrm{LV}$, with a more advanced disease and, more often, with the presence of overt heart failure $[8,22]$. In fact, our results show that patients with reduced TAPSE present thicker LV walls, enlarged left atria, more compromised LV diastolic function with increased left atrium filling pressure. Furthermore, they present more frequently significant mitral regurgitation, increased pulmonary artery pressure and advanced NYHA class.

Nevertheless our data also shows that troponin $(\geq 0.085 \mathrm{ng} / \mathrm{l})$, rather than NT-proBNP, has the best specificity and sensitivity in predicting RV dysfunction. However, the interpretation of those results may be misleading. Indeed, it should be kept in mind that NT-proBNP is almost exclusively removed through glomerular filtration and, therefore, renal insufficiency may lead to an additional increase of the peptide that is not to be attributed to amyloid heart involvement. Among the nine patients with NT-proBNP levels above the cut-off value of $2977 \mathrm{ng} / 1$, but without echocardiographic signs of RV dysfunction, eight showed high serum creatinine levels, with MDRD eGFR under the value of $50 \mathrm{ml} / \mathrm{min}$. Higher NT-proBNP cut-off values are probably required to identify RV dysfunction in amyloid patients with decreased eGFR [25]. On the other hand, only four patients without echocardiographic evidence of RV dysfunction showed troponin I serum levels above the cut-off value of $0.085 \mathrm{ng} / \mathrm{l}$, suggesting that this biomarker could be less influenced by the simultaneous presence of renal failure.
In $\mathrm{AL}$ amyloidosis, increased troponin I levels have been attributed to cardiac injury which could be mediated by several mechanisms, including microvascular coronary ischemia, mechanical effects of amyloid infiltration, and/or direct toxic effect of amyloid proteins and their precursors [26]. A recent study by Sied and coworkers demonstrated that global late gadolinium enhancement on cardiac magnetic resonance in patients with amyloidosis was associated with increased circulating troponin levels, suggesting a correlation of this biomarker with a more advanced stage of infiltration [27]. Therefore, the best performance of troponin I in predicting RV dysfunction could be explained by the fact that it is a marker of more diffused heart involvement.

\section{Study limitations}

The study presents all limitations of a cross-sectional design. Due to the small number of patients our observations must be considered preliminary and need to be confirmed in larger, prospective longitudinal trials that could explore further the relationship between NT-proBNP and troponin increase and the onset of RV dysfunction. The troponin assays are multiple, addressing either circulating troponin I or $\mathrm{T}$, with different sensitivity levels and with different imprecision levels. This could make the cut-off point proposed in the present study strictly associated with our series.

\section{Conclusion}

Our study demonstrates, for the first time, that a cut-off value of $2977 \mathrm{ng} / \mathrm{ml}$ for NT-proBNP and of $0.085 \mathrm{ng} / \mathrm{l}$ for troponin I are able to identify a subgroup of AL amyloidosis patients with RV dysfunction. Troponin I demonstrate better accuracy and proves to be the best biohumoral marker of $\mathrm{RV}$ involvement. Based on the results of the present study it is expected that simple measurements of cardiac biomarkers could easily and quite accurately suggest the presence of RV dysfunction, even when an accurate echocardiographic evaluation of right ventricular function is not feasible for anatomical or technical reason.

\section{Declaration of interest}

The authors report no conflicts of interest.

\section{References}

1. Merlini G, Bellotti V. Molecular mechanisms of amyloidosis. N Engl J Med 2003;349:583-96.

2. Perfetto F, Cappelli F, Bergesio F, Ciuti G, Porciani MC, Padeletti L, Moggi Pignone A. Cardiac amyloidosis: the heart of the matter. Intern Emerg Med 2013;8:191-203.

3. Rapezzi C, Merlini G, Quarta CC, Riva L, Longhi S, Leone O, Salvi F, et al. Systemic cardiac amyloidoses: disease profiles and clinical courses of the 3 main types. Circulation 2009;120:1203-12.

4. Koyama J, Ray-Sequin PA, Falk RH. Longitudinal myocardial function assessed by tissue velocity, strain and strain rate tissue Doppler echocardiography in patients with AL (primary) cardiac amyloidosis. Circulation 2003;107:2446-52.

5. Porciani MC, Lilli A, Perfetto F, Cappelli F, Massimiliano Rao C, Del Pace S, Ciaccheri M, et al. Tissue Doppler and strain imaging: a new tool for early detection of cardiac amyloidosis. Amyloid 2009;16:63-70.

6. Porciani MC, Cappelli F, Perfetto F, Ciaccheri M, Castelli G, Ricceri I, Chiostri M, et al. Rotational mechanics of the left ventricle in AL amyloidosis. Echocardiography 2010;27:1061-8. 
7. Bellavia D, Pellikka PA, Dispenzieri A, Scott CG, Al-Zahrani GB, Grogan M, Pitrolo F, et al. Comparison of right ventricular longitudinal strain imaging, tricuspid annular plane systolic excursion, and cardiac biomarkers for early diagnosis of cardiac involvement and risk stratification in primary systematic (AL) amyloidosis: a 5-year cohort study. Eur Heart J Cardiovasc Imaging 2012;13:680-9.

8. Cappelli F, Porciani MC, Bergesio F, Perlini S, Attanà P, Moggi Pignone A, Salinaro F, et al. Right ventricular function in $\mathrm{AL}$ amyloidosis: characteristics and prognostic implication. Eur Heart J Cardiovasc Imaging 2012;13:416-22.

9. Palladini G, Campana C, Klersy C, Balduini A, Vadacca G, Perfetti V, Perlini S, et al. Serum N-terminal pro-brain natriuretic peptide is a sensitive marker of myocardial dysfunction in $\mathrm{AL}$ amyloidosis. Circulation 2003;107:2440-5.

10. Dispenzieri A, Gertz MA, Kyle RA, Lacy MQ, Burritt MF, Therneau TM, Greipp PR, et al. Serum cardiac troponins and $\mathrm{N}$-terminal pro-brain natriuretic peptide: a staging system for primary systemic amyloidosis. J Clin Oncol 2004;22:3751-7.

11. Choi HS, Kim KH, Yoon HJ, Hong YJ, Kim JH, Ahn Y, Jeong MH, et al. Usefulness of cardiac biomarkers in the prediction of right ventricular dysfunction before echocardiography in acute pulmonary embolism. J Cardiol 2012;60:508-13.

12. Nagaya N, Nishikimi T, Okano Y, Uematsu M, Satoh T, Kyotani S, Kuribayashi $S$, et al. Plasma brain natriuretic peptide levels increase in proportion to the extent of right ventricular dysfunction in pulmonary hypertension. J Am Coll Cardiol 1998;31:202-8.

13. Gertz MA, Merlini G. Definition of organ involvement and response to treatment in $\mathrm{AL}$ amyloidosis: an updated consensus opinion. Amyloid 2010;17:48-9.

14. Levey AS, Bosch JP, Lewis JB, Greene T, Rogers N, Roth D. A more accurate method to estimate glomerular filtration rate from serum creatinine: a new prediction equation. Modification of Diet in Renal Disease Study Group. Ann Intern Med 1999;130:461-70.

15. Quinones MA, Otto CM, Stoddard M, Waggoner A, Zoghbi WA. Recommendations for quantification of Doppler echocardiography: a report from the Doppler Quantification Task Force of the Nomenclature and Standards Committee of the American Society of Echocardiography. J Am Soc Echocardiogr 2002;15:67-84.

16. Tei C, Ling LH, Hodge DO, Bailey KR, Oh JK, Rodeheffer RJ, Tajik AJ, et al. New index of combined systolic and diastolic myocardial performance: a simple and reproducible measure of cardiac function: a study in normals and dilated cardiomyopathy. J Cardiol 1995;26:357-66.

17. Rudski LG, Lai WW, Afilalo J, Hua L, Handshumacher MD, Chandrasekaran K, Solomon SD. Guidelines for the echocardiographic assessment of the right heart in adults: a report from the
American Society of Echocardiography: endorsed by the European Association of Echocardiography, a registered branch of the European Society of Cardiology and the Canadian Society of Echocardiography Inizio modulo. J Am Soc Echocardiogr 2010;23: 685-713.

18. Dispenzieri A, Kyle RA, Gertz MA, Therneau TM, Miller WL, Chandrasekaran K, McConnell JP, et al. Survival in patients with primary systemic amyloidosis and raised serum cardiac troponins. Lancet 2003;361:1787-9.

19. Dispenzieri A, Gertz MA, Kyle RA, Lacy MQ, Burritt MF, Therneau TM, Greipp PR, et al. Serum cardiac troponins and $\mathrm{N}$-terminal pro-brain natriuretic peptide: a staging system for primary systemic amyloidosis. J Clin Oncol 2004;22:3751-7.

20. Palladini G, Lavatelli F, Russo P, Perlini S, Perfetti V, Bosoni T, Obici L, et al. Circulating amyloidogenic free light chains and serum N-terminal natriuretic peptide type B decrease simultaneously in association with improvement of survival in AL. Blood 2006;107:3854-8.

21. Kumar S, Dispenzieri A, Lacy MQ, Hayman SR, Buadi FK, Colby C, Laumann K, et al. Revised prognostic staging system for light chain amyloidosis incorporating cardiac biomarkers and serum free light chain measurements. J Clin Oncol 2012;30: 989-95.

22. Ghio S, Perlini S, Palladini G, Marsan NA, Faggiano G, Vezzoli M, Klersy C, et al. Importance of the echocardiographic evaluation of right ventricular function in patients with $\mathrm{AL}$ amyloidosis. Eur J Heart Fail 2007;9:808-13.

23. Kim WH, Otsuji Y, Yuasa T, Minagoe S, Seward JB, Tei C. Evaluation of right ventricular dysfunction in patients with cardiac amyloidosis using Tei index. J Am Soc Echocardiogr 2004;17:45-9.

24. McMurray JJ, Adamopoulos S, Anker SD, Auricchio A, Böhm M, Dickstein K, Falk V, et al. ESC guidelines for the diagnosis and treatment of acute and chronic heart failure 2012: the Task Force for the Diagnosis and Treatment of Acute and Chronic Heart Failure 2012 of the European Society of Cardiology developed in collaboration with the Heart Failure Association (HFA) of the ESC. Eur J Heart Fail 2012;14:803-69.

25. Palladini G, Foli A, Milani P, Russo P, Albertini R, Lavatelli F, Obici L, et al. Best use of cardiac biomarkers in patients with $\mathrm{AL}$ amyloidosis and renal failure. Am J Hematol 2012;87:465-71.

26. Capone R, Amsterdam EA, Mason DT, Zelis R. Systemic amyloidosis, functional coronary insufficiency and autonomic impairment. Ann Intern Med 1972;76:599-603.

27. Syed IS, Glockner JF, Feng D, Araoz PA, Martinez MW, Edwards WD, Gertz MA, et al. Role of cardiac magnetic resonance imaging in the detection of cardiac amyloidosis. JACC Cardiovasc Imaging 2010;3:155-64. 